\title{
COMMISSION 14
}

\section{Atomic and Molecular data (Données atomiques et moléculaires)}

\section{Report of meetings on 24,25 and 31 July 1991}

$\begin{array}{ll}\text { President } & \text { : Sylvie Sahal-Bréchot } \\ \text { Vice President } & \text { : Wolfgang L. Wiese } \\ \text { Secretary } & \text { : Peter L. Smith }\end{array}$

Activities of Commission 14 at the XXIst General Assembly consisted in a business meeting session (August 24) and two scientific meetings: one open meeting of one session (August 31) and one specific interdisciplinary meeting "Astrophysical Opacities", of 4 consecutive sessions (August 25) complementary to that organized by Commission 35. In addition a Joint Commission meeting "Atomic and Molecular data for Space Astrophysics, needs and availability", was organized together with Commission 44, and sponsored by Commission 10 , $12,15,16,29,34,35$, and 36; it held on August 26, and is reported elsewhere.

\section{Business meeting (July 24, session 2)}

The President welcomed the members of the Commission and Peter L. Smith was appointed as Secretary.

\subsection{REVIEW OF MEMBERSHIP OF THE COMMISSION:}

Decease:

S. L. Mandelstam, Organizing Committee member.

Resignations:

Dr. F.Lovas resigned from the Organizing Committee.

Dr. A. Monfils resigned from Commission 14.

New members for Commission 14 (proposed by the President):

New IAU elect members:

Dr. Nicole F.Allard, Dr. Michèle Eidelsberg, Dr. Jean W. Gallagher, Dr.

Sydney Leach, Dr Jacques Le Bourlot, Dr Jean-Louis Lemaire, Prof.

Michael E. Mickelson, Dr. Annie Spielfiedel, Dr. Lydia Tchang-Brillet.

IAU members:

Dr Milan Dimitrijević, Dr. Hoang Binh Dy, Dr. Louis d'Hendecourt, Dr. David L. Lambert, Dr. François. Rostas, Dr. K. Sinha, Dr. Tran Minh Nguyet.

\subsection{PRESIDENT, VICE-PRESIDENT AND OFFICERS FOR THE TRIENNUM 1992-1995:}

After discussion, the new Organizing Committee for the triennum 1992-1995 was approved:

President: Dr. Wolfgang L. Wiese

Vice-President: Dr. William H. Parkinson

Members: $\quad$ Dr. Saül Adelman, Dr. Jacques Dubau, Dr. Alan H. Gabriel, Dr. Kato Takako, Prof. Ralph W. Nicholls, Prof. Harry Nussbaumer, Dr. Zenonas B. Rudzikas, Dr. Sylvie Sahal-Bréchot, Dr. Peter Smith. 


\title{
2.3. SCOPE OF THE COMMISSION AND WORKING GROUPS:
}

Dr. Jean Gallagher, who could only be appointed as a Consultant to Working Group 3 in the 1989-1991 triennum, since she was not a IAU member, was officially appointed as Chairperson of that Working Group.

The subject matter and the objectives of the Commission were rewiewed and discussed: the fertilization of the cross-discipline interaction between atomic and molecular physics is the major scope of the Commission. The increase of the need for a variety of atomic and molecular data in gaseous phase for astrophysics was noted, and thus the five preceding Working Groups were confirmed. In addition, owing to the increasing interest of solid phase chemistry (interaction gaz-surface), such as chemical reactions on grains, heterogeneous catalyse, photolyse, adsorption and desorption processes, it was decided to create a new Working Group on that topic; Sydney Leach was proposed to chair it; (contacted by the President after the General Assembly, he accepted the proposition).

Therefore the Working groups and their Chairmen for 1992-1995 were approved as follows:

1. Atomic spectra and wavelengths standards (W.C. Martin)

2. Atomic transition probabilities (W.L. Wiese)

3. Collision processes (J.W. Gallagher)

4. Line broadening (N. Feautrier)

5. Molecular structure and transition data (W.H. Parkinson)

6. Molecular reactions on solid surfaces (S. Leach)

$* *$

\section{Astrophysical Opacities (July 25, sessions 1-4)}

Scientific Organizing Committee: A.E. Lynas-Gray, A.K. Pradhan, M.J. Seaton, C.J. Zeippen. INTRODUCTION - Chair: W.L. Wiese

\section{STELLAR ENVELOPE OPACITIES}

\author{
F. J. ROGERS
}

Lawrence Livermore National Laboratory, P.O. Box 808, Livermore, California 94550, USA

Until recently most studies of stellar evolution and pulsation have relied on opacities calculated at Los Alamos National Laboratory in the 60's and 70's; prior to the advent of supercomputers. Consequently, a number of approximations were made to facilitate calculational tractibility. For example, they used of hydrogenic oscillator strengths and neglected configurational term splitting in bound-bound transitions. As observational capabilities increased, a number of substantial disagreements with theoretical prediction were noted. Several studies found that increasing the opacity in specific temperature regions could reduce or eliminate these disagreements (Stellingwerf, 1978; Simon, 1982). This inspired Norman Simon to call for new independent calculations of opacity. Several groups have responded to this plea and a in depth discussion of these efforts will be presented in this session. In addition, results from some recent, very encouraging, studies that have used the new opacities will be presented.

Stellingwerf, R.F, 1978, Astron.J., 83, 1184

Simon, N. R., 1982, Ap. J. (Letters), 260, L87

\section{MOLECULAR OPACITIES}

The introductory remarks on molecules are reported along with the following report on the talk about molecular data presented by U. G. Jorgensen. 
2.1. SESSION 1. ATOMIC AND MOLECULAR DATA - Chair: W.L. Wiese

\author{
R-MATRIX METHODS FOR CALCULATION OF ATOMIC RADIATIVE DATA \\ M.J SEATON ${ }^{*}$, A.K. PRADHAN ${ }^{+}$and J.A. TULLY ${ }^{+}$ \\ * : University College London, Gower Street, London WCIE 6BT, U.K. \\ + : Department of Astronomy, Ohio State University, Columbus, Ohio 43210,USA \\ $t$ : Observatoire de la Côte d'Azur, URA CNRS 1362, B.P. 139, O6003 Nice CEDEX, France
}

\title{
1. General Description (Seaton)
}

The Opacity Project (OP) is an international collaboration (see Seaton et al., 1991) concerned with the calculation of opacites and of the required atomic data. I will give a general description of the methods used in the atomic-physics work and two of my colleagues will give some examples of results obtained. Let me first recapitulate some basic ideas of atomicstructure theory.

- The Central-Field (CF) model. An electron in a central field has a wave function $\phi_{n l m}=Y_{l m}(\hat{\mathbf{r}})(1 / \mathrm{r}) \mathrm{P}_{n l}(\mathbf{r})$.

- Configurations. A configuration is defined by a set of $n l$ quantum numbers and has a wave function $\Phi\left(x_{1}, \ldots, x_{N}\right)$ constructed using functions $\phi_{n l m}$ and anti-symmetrised in spaceand-spin coordinates $x_{i}=\left(\hat{r}_{i}, \sigma_{i}\right)$.

- Configuration Interaction (CI). The wave functions are $\Psi_{k}=\Sigma_{j} \Phi_{j} c_{j k}$ with the coefficients $c_{j k}$ such as to diagonalise $\left(\Psi_{k}|H| \Psi_{k^{\prime}}\right)$.

- Close-Coupling (CC). The wave functions are $\Psi=\$ \Sigma_{i} \psi_{i}\left(x_{i}, \ldots, x_{N}\right) \theta_{i}\left(x_{N+1}\right)$ where $\psi_{i}$ is a $\mathrm{CI}$ function for an N-electron "target" state, $\theta_{i}$ a function for an added electron and $\$$ an anti-symmetrisation operator.

In the OP work the $\mathrm{CI}$ and $\mathrm{CC}$ approaches are combined, the wave functions being $\Psi=\Sigma_{i} \psi_{i}\left(\theta_{i}\right)+\Sigma_{j} \Theta_{j} c_{j}$. The functions $\theta_{i}$ and coefficients $c_{j}$ are optimised using R-matrix methods (Berrington et al., 1987). Calculations of energy-levels, $f$-values and photoionisation cross sections have been made for all cosmically abundant elements in all ionisation stages, and for all excited states up to $n=10$. Availability of the atomic data is discussed by Mendoza (1991).

For systems containing up to 12 electrons the calculated f-values and photoionisation cross sections should be accurate to within about $10 \%$ (except for transitions involving delicate cancellations). For the more complex systems the data may be less accurate (due to truncations in the expansions) and less complete (some supplementary data have been calculated using more approximate methods). Methods used for the calculation of line profiles are described by Seaton (1987a, 1987b, 1988, 1989, 1990).

\section{Photoionisation of Fe II (Pradhan).}

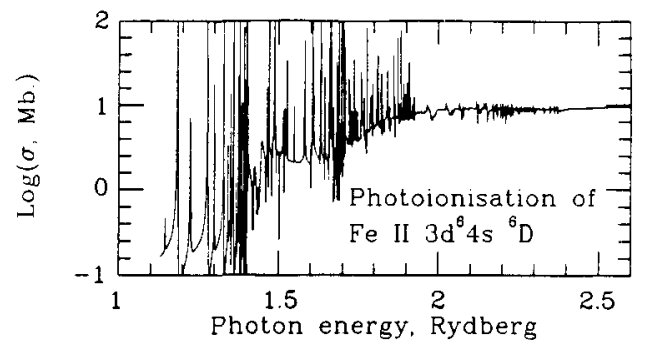

Some of the most OP extensive calculations have been carried out for the ions Fe I - VI. As an example consider the (electron $+\mathrm{Fe}$ III) system to obtain oscillator strengths and photoionization cross sections for Fe II. In Fig. 1 we present the photoionization cross section for the ground state $3 d^{6}(5 \mathrm{D}) 4 s^{6} \mathrm{D}$, including 21 Fe II target states (Le Dourneuf et al., 1991).

Figure 1 


\section{Results for Be-like ions (Tully)}

We have computed energy levels, oscillator strengths and photoionisation cross sections for 15 members of the Be sequence. Jannitti et al. (1986) have measured photoionisation of $\mathrm{B}^{+}$ $2 s^{2}{ }^{1} \mathrm{~S}$ and $2 s 2 p^{3} \mathrm{P}^{\circ}$. Their threshold cross-sections are in quantitative agreement with our data and their resonances in the ${ }^{1} \mathrm{P}^{\circ}$ continuum resemble those we predict. Lang et al. (1987) have performed lifetime measurements in N IV, O V and Ne VII. Six of their $15 A$ values agree with ours to better than 10 per cent. But there are big differences for 7 transitions with fairly small $A$ values. The OP results agree however with those from independent CI calculations (see Tully et al. 1990). Fawcett (1984) has calculated gf values for many ions in the sequence, taking into account spin-orbit coupling and CI. For some transitions our results are quite close to his while for others they disagree by more than 50 per cent. (see Tully et al. 1991 and Seaton et al. 1991). One possible explanation is the breakdown in LS coupling.

\section{$\underline{\text { References }}$}

K.A. Berrington, P.G. Burke, K. Butler, M.J. Seaton, P.J. Storey, K.T. Taylor and Yu Yan. J. Phys. B., 20, 6379, 1987.

M. Le Dourneuf, S.N. Nahar and A.K. Pradhan, J.Phys.B (to be published in J.Phys.B).

B.C. Fawcett, At. Data Nucl. Data Tables, 30, 1, 1984; erratum 33, 479, 1985.

E. Jannitti, F. Pinzhong and G. Tondello, Phys. Scr. 33, 434, 1986.

J. Lang, R.A. Hardcastle, R.W.P. McWhirter and P.H. Spurrett. J. Phys. B., 20, 43, 1987.

C. Mendoza. IAU 21st General Assembly, Joint Commission Meeting on "Atomic and Molecular Data for Space Astronomy"

M.J. Seaton, J. Phys. B, 20, 6363, 1987a; 20, 6431, 1987b; 21, 3033, 1988; 22, 3603, 1989; 23, 90, 1990.

M.J. Seaton, C.J. Zeippen, J.A. Tully, A.K. Pradhan, C. Mendoza, A. Hibbert and K.A. Butler. Proceedings of Workshop on Astrophysical Opacities (Caracas, July 1991), to be published in Revista Mexicana de Astronomia y Astrofisica.

J.A. Tully, M.J. Seaton and K.A. Berrington. J. Phys. B., 23, 3811, 1990.

J.A. Tully, M.J. Seaton and K.A. Berrington. J. Phys. (Paris) IV, C1, Suppl. J. Phys. II, 1, 169, 1991.

\section{MOLECULAR DATA \\ Uffe Gråe JØRGENSEN \\ Niels Bohr Institute, Blegdamsvej 17, DK-2100 Copenhagen, Denmark}

The most common uses of molecular data for astrophysical purposes are probably identification of spectral features, determination of isotopic ratios, abundance determination, and computation of stellar model atmospheres. For the two first purposes information about only the lines of interest may sometimes be sufficient, and of the order half a million lines are known from laboratory measurements today. This is, nevertheless, only a tiny fraction of the line-data needed for the two latter purposes, but it would of course be meaningless to try to measure all the lines, and good methods that can combine molecular constants from laboratory experiments with quantum mechanically computed frequencies and intensities have also been developed. The advantage of the ab initio method is that it can handle a complete computation of all lines up to the dissociation energy of the molecule. The disadvantage is that it generally does not allow for an internal uncertainty estimate. Tight interaction between theoretical predictions and laboratory experiments is therefore crucial. Preferably, data for all molecules of interest should fulfill the following four criteria.

1) The strongest bands to a certain limit should be measured with good accuracy in the laboratory to assure that the absorption coefficient integrated over the whole spectrum is correct, and that the lines easily visible in stellar spectra can be traced accurately.

2) The complete set of lines up to the dissociation energy should be calculated to assure that the right effect on the model atmospheric structure and low resolution spectra (including the continuum drawing) can be predicted. 
3) A sample of very weak bands and lines that include high energy upper or lower states should be measured in the laboratory and compared with the computations to test the quality of the most uncertain part of thecalculated potential and dipole moment functions.

4) The temperature dependence of the absorption coefficient in low resolution should be measured to ensure that the computed integrated absorption coefficient has the right temperature dependence.

These criteria are not fulfilled for any of the molecules we are interested in, but hopefully a greater collaborative effort will allow this. Point 4) above is only fulfilled for water, point 3) only for $\mathrm{HCN}$, and point 2) only described for $\mathrm{CN}$, whereas point 1) is fulfilled for most astrophysically important molecules except some of the radicals. The expected wealth of data from the coming satellites with high resolution spectroscopic capacity in the infrared will be hard to use satisfactorily without a considerably better astrophysical molecular data base.

The most extensive compilations of empirical data are the HITRAN (L.S. Rothman et al. 1987; Applied Optics, 26, 4058) and the GISA (L. Husson et al. 1986, Ann. Geophys., 4A, 185) data bases. The latest released edition of the HITRAN data base contained strength, frequency and excitation energy for 348,000 lines from 28 molecules (70 isotopic varieties), and the coming release is expected to contain more than 600,000 lines. The GISA data base contain basically the same data as the HITRAN, plus a few extra molecules of mainly environmental interest, and the next release is expected to contain slightly more than 700,000 lines in total. Laboratory data on lines from a smaller number of molecules (including $\mathrm{TiO}, \mathrm{ZrO}, \mathrm{YO}, \mathrm{CN}$, $\mathrm{FeH}$ and $\mathrm{LaO}$ ) of particular astrophysical interest, are available from J.G. Phillips and S.P. Davis (1987, Publ. Astron. Soc. Pac., 99, 1105; and preceeding research).

Line strength, line frequency andexcitation energy are listed for a total of 22 million lines of 20 diatomic molecules and 35 million lines of 4 polyatomic moleculesin the astrophysical data bases. Only little is known about the quality of the data for the weak lines and bands, and no comparison of data computed by different groups exists.

R.L. Kurucz (1991; in Stellar Atmospheres: Beyond Classical Models, eds. Crivellari et al., NATO ASI, p.421) has for many years offered a compilation of molecular lines to the community, which now contain 15.7 million lines from the molecules $\mathrm{H}_{2}, \mathrm{CH}, \mathrm{NH}, \mathrm{OH}, \mathrm{MgH}$, $\mathrm{SiH}, \mathrm{CO}, \mathrm{SiO}, \mathrm{CN}, \mathrm{C}_{2}, \mathrm{TiO}$, in various isotopic varieties.

U.G. Jorgensen and M. Larsson (1990, Astron. Astrophys., 238, 424) computed a line list of 3.3 million lines from the "red" (A-X) system of CN. The list is the first to include a completeness study, in the sense that the Boltzman factor of the energy levels contribute more than $99 \%$ of the theoretical partition function for all temperatures of astrophysical interest. The haze of weak lines was found to influence the low-resolution synthetic spectra substantially. The line list is available on request together with similar lists for $\mathrm{CH}$ (in preparation), $\mathrm{H}_{2} \mathrm{O}$ (in preparation) and $\mathrm{HCN}$ (up to 10 million lines; U.G. Jørgensen et al. 1985, J. Chem. Phys., 83, 3034).

D.R. Alexander et al. (1989, Astrophys. J., 345, 1014) computed, and offers, a list of 4.5 million lines from the electronic ground state of the water molecule. The method developed by the authors use observed lines to define a statistical distribution function of line strengths and lower excitation levels, and assume pre-specified values of line spacing and number of lines to distribute observed low resolution absorption data into individual lines. The observational low resolution data is available from C.B. Ludwig et al. (1973, Handbook Infrared Rad. Combustion Gases, NASA SP-3080).

J.M. Brett (1990, Astron. Astrophys., 231, 440) estimated the f-values of the A-X and B-X system of $\mathrm{VO}$ and the $\mathrm{f}$-value of the TiO $\varepsilon$ system, as the ratio between the intensity of these bands and the band from TiO $\delta$ in observed stellar spectra, and offers a tape with 2 million lines from the two VO systems. 
F. Querci et al. (1974, Astron. Astrophys., 31, 265) compiled, and offers, a tape with gf-values, frequencies and excitation energies (but only identification of which molecule and isotope each line belongs to) of one million lines from $\mathrm{CN}, \mathrm{C}_{2}$ and $\mathrm{CO}$.

J.E. Littleton and S.P. Davis (1985, Astrophys. J., 296, 152) measured f-values of bands from $\mathrm{ZrO}$ and $\mathrm{YO}$, and calculated from this (in yet unpublished work) the frequency, strength and excitation energy for $332,000 \mathrm{ZrO}$ lines and 1,200 YO lines. The data are available from J.E. Littleton on request.

A.L. Piñeiro et al. (1987, J. Mol. Spec., 125, 184; and references therein) offers a compilation of 14,000 lines of SiO, 35,000 lines of CO, 35,000 lines of CS, and 600 lines of SiS. Their method has been to use dipole moment computations from the literature and empirical data to fit a Pade approximant to the dipole moment function, and from this calculate all transitions within what they consider the well determined part of the dipole moment function.

\subsection{SESSION 2: EQUATIONS OF STATE - Chair: A.K. Pradhan}

\section{EQUATIONS OF STATE: COMPARISONS OF RESULTS FROM TWO COMPETING FORMALISMS. \\ Werner DÄPPEN \\ Departments of Physics and Astronomy, University of Southern California, Los Angeles, USA}

Currently there are two basic approaches to the equation of state for stellar envelopes and interiors. Both want to realize the same goal, equilibrium statistical mechanics of reacting plasmas, but their methods differ. One (the older) method chooses the so-called "chemical picture", in which the notion of atoms is maintained despite the plasma environment. A mixture of atoms, molecules, ions, electrons and nuclei is considered, and the occurring ionization and dissociation reactions (thus the name chemical picture) are treated according to the entropy-maximum (or free-energy minimum) principle, which is, in the language of the astrophysicists, the Saha equation. Interactions of the plasma with atoms and ions are introduced separately in a heuristic way. The other (more recent) method is based on the socalled "physical picture", where only fundamental particles (electrons, nuclei) explicity enter. Furthermore, through the means of activity expansions, the problems of plasma physics and statistical mechanics are treated on the same footing.

The international "Opacity Project" (OP) uses an equation of state realized in the chemical picture (Mihalas, Hummer, Däppen , MHD), and the opacity effort pursued at Livermore by Iglesias and Rogers (OPAL) one in the physical picture (hereinafter Livermore equation of state). Since the equation of state plays an important role in any opacity calculation, because it predicts both level populations and ionization degrees, and since at the desired level of accuracy no laboratory experiments exist, comparisons of theoretical formalisms are so far the only means to estimate the source of opacity uncertainty due to the equation of state.

First comparisons concentrated on the case of conditions as found in the H-He ionization zones of the Sun. For both equations of state, pressure and thermodynamic quantities were compared with those of simple mixtures of ideal gases, whose ionization equilibrium is determined by a Saha equation that contains ground states only. It was found that in these regions of the Sun, the predicitons of the MHD and Livermore equations of state are virtually the same, despite the fact that they differ from the simple Saha results by a few percent $[1,2]$.

This agreement was by no means expected, and it turned out that it was due to the Coulomb interaction, present in the MHD and Livermore equation of state, which is the dominating non-ideal effect under these conditions. And yet, despite the common Coulomb treatment, one would intuitively expect a greater difference, essentially because of the treatment of the excited states of atoms and ions in the MHD equation of state. That this is not the case could indicate a 
perhaps accidental cancellation of contributions within the MHD formalism, and the question is currently being examined.

Very recently, Forrest Rogers and I again compared results, this time for higher temperatures and densities $\left(p=10^{-2} \ldots 10 \mathrm{~g} / \mathrm{cm}^{3}\right)$ [3]. We found a good agreement for solar conditions, that is for the temperatures as they are in the Sun at these densities. However, the Sun just marginally passes: for slightly less massive stars, the discrepancy soon becomes very important indeed. Finally, we also considered the case of heavier elements. Here, for the first time we succeeded in establishing a clear case of disagreement between the MHD and Livermore results. For a solar composition, at a temperature of about $210^{5} \mathrm{~K}$ and a density of 5 $10^{-3} \mathrm{~g} / \mathrm{cm}^{3}$, the predicted ionization degrees of $\mathrm{C}, \mathrm{N}$ and $\mathrm{O}$ are quite drastically different, with the Livermore values lying closer to the simple Saha results. Despite the fact that of course only some $2 \%$ of the matter in the Sun consists of elements heavier that $\mathrm{H}$ and $\mathrm{He}$, the present state of helioseismology carries the potential of direct thermodynamic tests (via sound speed) of the equation of state under these conditions [4]. Furthermore, since there are (though quite localized) zones with distinctly different ionization degrees (by up to a factor of 2), opacity will undoubtedly also be influenced by this equation of state issue, and stellar cases might be found that could test the equation of state via the opacity.

[1] W. Däppen, Y. Lebreton, F. Rogers, 1990: Solar Physics , 128, 35-47.

[2] W. Däppen, 1990: in "Progress of seismology of the sun and stars" (eds. Y. Osaki \& H. Shibahashi, Springer)

[3] W. Däppen , to appear in the proceedings of the workshop "Astrophysical Opacities" (Caracas, July 1991)

[4] J. Christensen-Dalsgaard, W. Däppen : 1992, Astronomy and Astrophysics Review.

\section{MOLECULAR EQUILIBRIA WITH CONDENSATION IN ASTROPHYSICAL GASES} C.M. SHARP

Service P.T.N., Centre d'Etudes de Bruyères-le-Châtel, BP 12, F-91680 Bruyères-le-Châtel, France

\section{Introduction}

In gases at temperatures below about $6000 \mathrm{~K}$, molecules play an important rôle in the opacity, and below about $2000 \mathrm{~K}$ grain scattering can increase the opacity very much further. These conditions are very typical of stars and planets during their formation processes, together with brown dwarfs and circumstellar shells. If thermodynamic equilibrium is assumed, the abundances depend on the element composition and the temperature and pressure. However, most previous work on molecular equilibria examined the effects of temperature and pressure, with at best only a few compositions.

As is well known, the abundances of the molecular species can depend in a very sensitive and non-linear way on the element composition, particularly the $\mathrm{C} / \mathrm{O}$ ratio due to $\mathrm{CO}$ being the most tightly bound of all diatomic molecules, together with the presence of any condensed species. In order to reduce the parameter space to two dimensions, we have calculated molecular abundances for a large number of temperatures and $\mathrm{C} / \mathrm{O}$ ratios for a fixed total pressure, with condensates included when appropriate. Only the gas phase and how it is modified by condensed phases is discussed here.

\section{Discussion of Calculations}

73 mixtures with compositions of $\log (\mathrm{C} / \mathrm{O})=-0.37$ to 0.35 in steps of 0.01 were computed in one degree intervals from 4000 to 1000 at a constant total pressure of $500 \mathrm{dyne} / \mathrm{cm}^{2},\left(4.935 \times 10^{-4}\right.$ atmospheres). Each mixture had the 20 most abundant elements with, excepting carbon, the Anders and Grevesse (1989) solar mixture with suggested modifications by Meyer (1991). For the different mixtures only carbon was changed, with the ratio of all other elements with respect to hydrogen being kept at the solar values. For the solar mixture $\log (C / O)=-0.33$. 
The gas phases consisted of the 20 neutral atoms, and 68 diatomic and 74 polyatomic molecules. Additionally above $2000 \mathrm{~K}, 39$ charged species consisting of free electrons, and atomic and molecular ions, were included, and below $2000 \mathrm{~K}, 54$ condensed species were specified, of which a proportion, depending on temperature and composition, would appear in the condensed phases. The most important condensates were the oxides, silicates, carbides and graphite, and included in the calculations was a solid solution of melilite $\left(\mathrm{Ca}_{2} \mathrm{Al}_{2} \mathrm{SiO}_{7}\right.$ and $\mathrm{Ca}_{2} \mathrm{MgSi}{ }_{2} \mathrm{O}_{7}$ ).

Most of the thermodynamic data were obtained from the JANAF tables (Chase, 1982), which were put in a form suitable for computation. For each temperature and composition, the abundances of each species in the gas phase, together with the condensates below $2000 \mathrm{~K}$, were obtained by minimising the total Gibb's free energy of the system, together with the constraints of mass balance and total charge neutrality, using the computer code SOLGASMIX (Besmann, 1977), which we have substantially improved (Sharp and Huebner, 1990).

\section{Results}

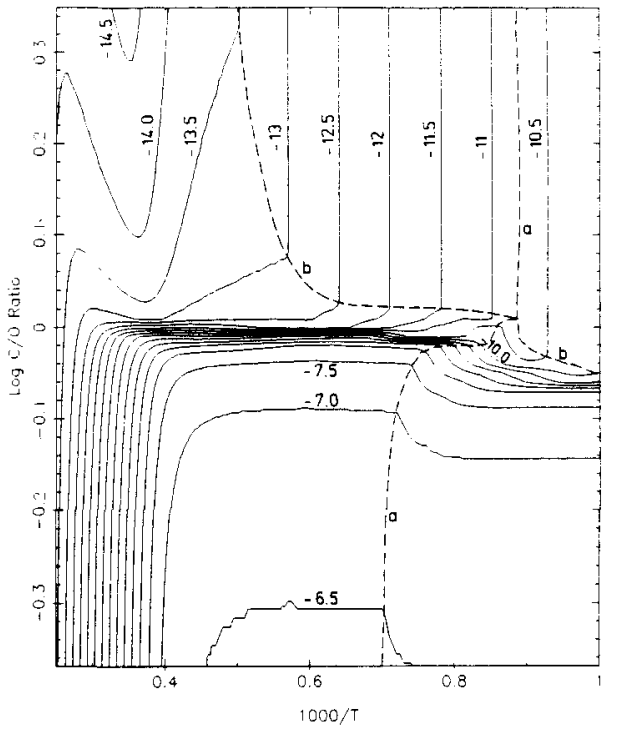

Fig. 1 Contours of log partial pressures in atmospheres of $\mathrm{H}_{2} \mathrm{O}$ for different temperatures and compositions at a constant total pressure of $4.935 \times 10^{-4}$ atmospheres. The dashed curves "a" and "b" mark the high temperature limits of the condensates $\mathrm{Mg}_{2} \mathrm{SiO}_{4}$ and graphite respectively.
Figure 1 is a contour plot of the log partial pressure of $\mathrm{H}_{2} \mathrm{O}$, which can be a particularly important opacity source at low temperatures, in atmospheres as a function of $\log (\mathrm{C} / \mathrm{O})$ and $1000 / \mathrm{T}$. It is seen that for small changes of the $\mathrm{C} / \mathrm{O}$ ratio in the region where that ratio is close to unity, the abundance of $\mathrm{H}_{2} \mathrm{O}$ changes very rapidly, and is due to $C O$ controlling the chemistry of the gas phase At higher temperatures $\mathrm{H}_{2} \mathrm{O}$ dissociates, and when the temperature is decreased for the oxygen rich case the abundance also falls due to oxygen being taken out of the gas phase by the condensation of oxides and silicates. The most important sink of oxygen is the condensate $\mathrm{Mg}_{2} \mathrm{SiO}_{4}$, which appears to the right of the dotted curve "a".

In the carbon rich case the increase in the $\mathrm{H}_{2} \mathrm{O}$ abundance with decreasing temperature is due to carbon being taken out of the gas phase mostly by graphite, this reduces the abundance of $\mathrm{CO}$ which frees up some oxygen that can form $\mathrm{H}_{2} \mathrm{O}$. The vertical contours are equally spaced and reflect the vapour pressure curve of graphite, which appears to the right of the dotted curve " $b$ ". Note that in this region curve "a" no longer plays an important rôle.

Plots of many molecules containing carbon, such as $H C N$, are almost complementary to $\mathrm{H}_{2} \mathrm{O}$ with the maxima and minima interchanged. The effects of condensation on $\mathrm{HCN}$ abundances for different compositions were discussed in Sharp (1990).

\section{Conclusions}

We have shown here that the abundance of $\mathrm{H}_{2} \mathrm{O}$ is very sensitive to the $\mathrm{C} / \mathrm{O}$ ratio when that ratio is close to unity in the temperature range considered, and for certain conditions the abundance can also be sensitive to the formation of condensed species. This region separates 
areas where the abundance is relatively insensitive to changes of temperature and composition, and is seen for many other molecules. This will be the subject of a more detailed future paper.

\title{
$\underline{\text { References }}$
}

Anders, E. and Grevesse, N.: 1989, Geochim. Cosmochim. Acta, 53, 197.

Besmann, T.M.: 1977, "SOLGASMIX-PV, A computer Program to calculate Equilibrium Relationships in Complex Chemical Systems"(Oak Ridge National Laboratory Report TM5775).

Chase, M.W.: 1982, JANAF Thermodynamic Tables, Magnetic Tape Version (Midland, MI: Dow Chemical Co.).

Meyer, J.-P.: 1991, Private communication.

Sharp, C.M.: 1990, Astrophysics and Space Science, 171, 185.

Sharp, C.M. and Huebner, W.F.: 1990, Ap. J. Suppl. Ser., 72,417.

\subsection{SESSION 3. OPACITIES - Chair: T.R. Carson}

\author{
RADIATIVE ATOMIC ROSSELAND MEAN OPACITY TABLES \\ Carlos A. IGLESIAS and Forrest J. ROGERS \\ Lawrence Livermore National Laboratory, P.O. Box 808, Livermore, California 94550, USA
}

We present radiative Rosseland mean opacity tables calculated with the OPAL code (Iglesias and Rogers 1991) which removes several approximations present in previous calculations. In particular, improvements in the atomic physics have been incorporated which yield reasonably accurate photon absorption data (accuracy comparable to single-configuration, selfconsistent field calculations with relativistic corrections) and include the configuration term splitting in the LS-coupling scheme. In some cases, the latter can increase the number of spectral lines by orders of magnitude compared to previous opacity codes. OPAL also employs an equation of state method which avoids the usual ad hoc cut offs introduced in free energy minimizaton schemes. The opacity tables are given in terms of temperature and $R$, where $R=$ $\rho / T^{3}$ with $\rho$ the density and $T$ the temperature. Extensive results are given for the AndersGrevesse (1989) metal abundances which allow accurate interpolation in temperature, density, hydrogen mass fraction, and metal mass fraction. The range of $T$ and $R$ cover typical stellar conditions from the interior to the hotter atmospheres. Cool atmospheres are not considered since photoabsorption by molecules is neglected. Only radiative processes are taken into account so that electron conduction is not included.

Comparison to the Los Alamos Opacities (Huebner 1986; Cox and Tabor 1976) show factors of 2-3 opacity enhancements at temperatures around $300,000 \mathrm{~K}$, which are mostly due to the improved atomic physics of partially filled M-shell ions. As a result, the OPAL opacities show considerably more sensitivity to the metallicity than earlier calculations. Uncertainties in this new opacity bump near $T=300,000 \mathrm{~K}$ have been investigated. It is found that several issues such as line broadening, the angular momentum coupling in the atomic data generation, and metal composition can significantly affect the results. More modest opacity enhancements are reported $(\sim 20 \%)$ at 3 million degrees which are due to the improved atomic data at lower densities and to the equation of state at the higher densities. Not all differences result in increased opacities. Reductions of $\sim 20 \%$ are found in the region $10,000 \mathrm{~K}<T<100,000 \mathrm{~K}$. These opacity decreases have been traced to the treatment of the hydrogen Lyman- $\alpha$ line wing. There are also opacity decreases at the highest temperatures and densities $(\sim 15 \%)$ where differences in the treatment of degeneracy affect the photon scattering from free electrons.

\section{$\underline{\text { References }}$}

Anders, E. and Grevesse, N. 1989, Geochim. Cosmochim.Acta. , 53, 197

Cox, A. N. and Tabor, J. E. 1976, Ap. J. Suppl. , 31, 271 
Iglesias, C. A. and Rogers, F. J.1991, Ap. J. (Letters), 371, L73

Huebner, W. F. 1986, in Physics of the Sun, ed. P. A. Sturrock, T. E. Holzer, D. M. Mihalas, and R. K. Ulrich (Dordrecht: Reidel), 1, 33

\section{THE OPACITY PROJECT: OPACITIES YU YAN}

Department of Astronomy, University of Illinois, 1002 West Green Street, Urbana, Illinois 61801, USA.

\section{Introduction}

The current interests in revisions of stellar opacities stem largely from a study by Simon (1982), who suggested that the heavy element contribution to the opacity might have been under-estimated by a factor of 2--3 in previous opacity calculations (for example, Cox and Tabor 1976). In 1983 a number of researchers in atomic physics and astrophysics came together to discuss a joint effort to recalculate stellar opacities; parallel works on the equation of state, line broadening theories and streamlined production of accurate atomic data were pursued at various research centres in five countries. This was later known as the Opacity Project (OP; see, for example, Seaton 1987). Another major effort at reexamining heavy element opacities is the OPAL project (Iglesias, Rogers and Wilson 1987, 1990). Here we discuss briefly the methods used in the OP calculation of stellar opacities, and present selected results in a format for easy comparison with those of OPAL.

\section{Methods and selected results}

We use the MHD equation of state (Hummer and Mihalas 1988, Mihalas, Däppen and Hummer 1988). It is based on the free energy minimisation method, and takes into account non-ideal effects due to various plasma interactions. The chemical picture assumed by this equation of state is especially suited to opacity calculations, as the relevant spectroscopic notions find a direct equivalence in the formalism.
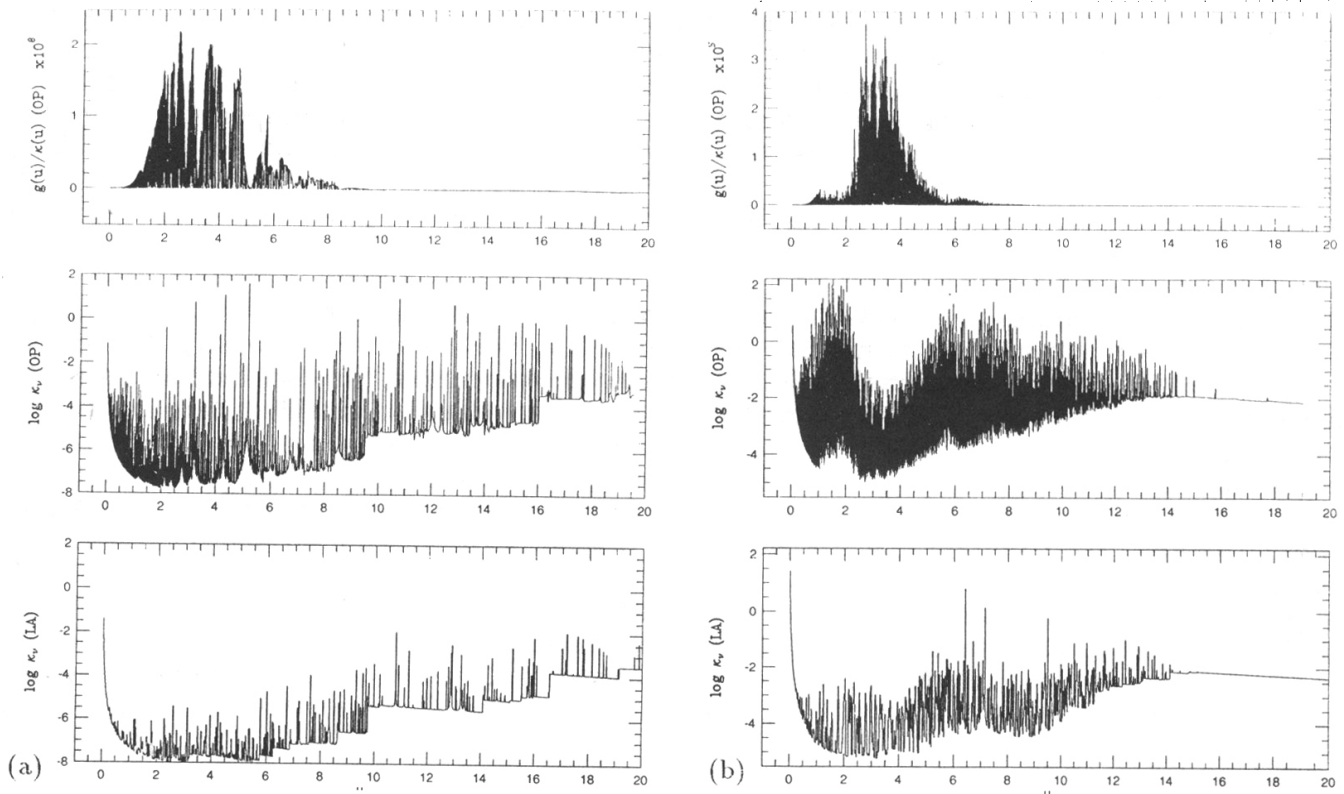

Fig. 1.- Monochromatic opacities from LAAOL and OP (lower, middle plots respectively) and the corresponding OP Rosseland integrand (upper plot) for (a) pure carbon at $\log T=4.4626, \log \rho=8.033$ and (b) pure iron at $\log T=5.5417, \log \rho=-5.241$. 
We use close-coupling type atomic data, supplemented at high target energies by a "top-up" procedure (see the paper by M.J. Seaton in this volume). Figure 1(a) compares pure carbon monochromatic opacities at one $(T, \rho)$ tabulated in the Los Alamos Astrophysical Opacity Library LAAOL; see Hüebner et al. 1977), lower plot, to those obtained using the OP method, middle plot. The effect of including detailed auto-ionisation features on the mean opacity is most clearly seen in the Rosseland integrand of the OP spectrum, upper plot. The corresponding Rosseland means are 3.16 (LAAOL) and $6.76(\mathrm{OP}) \mathrm{cm}^{2} / \mathrm{g}$. Figure $1(\mathrm{~b})$ compares the pure iron monochromatic opacities at another $(T, \rho)$. The higher opacities obtained by the OP can mostly be attributed to the much improved atomic model.

The OP atomic data and the opacity codes have undergone extensive checks; several full tables of mean opacities were produced in the process. Figures $2(a, b)$ compare the OP and OPAL Rosseland means for $X=0.7, Z=0.02$ and "AG abundances" (Anders and Grevesse 1989) at a range of temperatures and two values of the density parameter $R=\rho / T_{6}{ }^{3}$, where $T_{6}$ is temperature in $10^{6} \mathrm{~K}$. The current agreement between the two calculations is in general quite good; some rather significant discrepancies develop at low densities and temperatures above $10^{5} \mathrm{~K}$ (the so-called "Z-bump"), the source of which is yet to be determined. We note, however, that the OP results may undergo upward revisions in the near future, due mainly to inclusion of the following effects: (i) fine structure and intermediate coupling, (ii) transitions involving highly excited target states, (iii) ions of nickel. For a fuller discussion of future revisions in stellar opacity calculations see Yu Yan (1991).

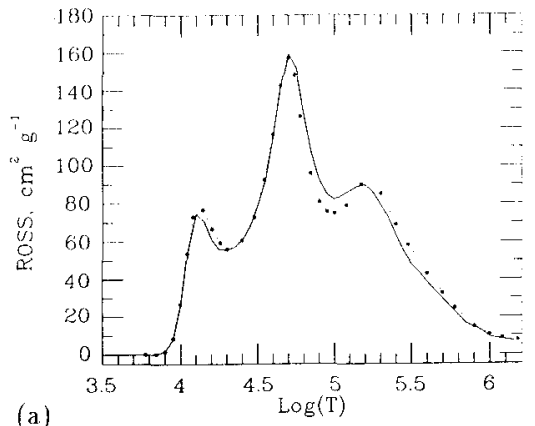

(a)

Fig 2 -Rosseland mean opacities for $X=0.7, Z=0.02$ (b) $\frac{\text { Fig. 2.-Rosseland mean opacities for } X=0.7, Z=0.0}{\log R=-3.5 . \text { Curves are: } 2 \text { OP }}$

\section{The Opacity Library}

The many man-years of research effort committed to this work will culminate in an Opacity Project User Service (OPUS), which will provide a user-oriented, publicly accessible opacity library for the astrophysical community, capable of computing mean and multi-group mean opacities, opacity distribution functions and monochromatic opacities of arbitrary composition. The design of the opacity library has been made simple by the approximation, which was verified to be sufficiently good, that the statistical-mechanical properties of a chemical element is independent of the plasma composition at a given electron density $N_{e}$. In practice we have computed and archived single element monochromatic opacities using occupation numbers from the solar mixture on the grid $3.5 \leq \log T \leq 7.2, \Delta \log T=0.025$, $\Delta \log N_{e}=0.25$ and lower, upper bounds for $\log N_{e}$ varying with $\log T$. For this grid and 14 chemical elements the amount of monochromatic opacity data after packing is about 12 gigabytes. Mixing codes have been developed to construct mixture opacities from the archive of single element monochromatic opacities. The archive and the mixing codes form the Opacity Library. 
The production of mixture opacities from the Opacity Library is computationally straightforward. This simplified mixing scheme is the basis for OPUS, which will evolve in several stages towards increasing autonomy and ease of use. Given the rapid advances in workstation technology, it is hopeful that a stand-alone, locally maintained facility may soon provide the entire resources for OPUS, allowing all manners of network access.

\title{
Acknowledgment
}

Other members of the OP involved in the opacity calculations are D.M. Mihalas, A.K. Pradhan and M.J. Seaton.

We thank F.J. Rogers and C.A. Iglesias for providing data for comparison in advance of publication.

Work supported by National Science Foundation grants AST85-19209 and AST89-14143, and by unrestricted research funds from the University of Illinois.

\section{References}

Anders, E. and Grevesse, N. 1989, Geochim. Cosmochim Acta., 53, 197.

Cox, A.N. and Tabor, J.E. 1976, AP. J. Supp., 31, 271.

Hüebner, W.F., Merts, A.L., Magee, N.H.Jr. and Argo, M.F. 1977, Astrophysical Opacity Library, Los Alamos Scientific Laboratory Report LA-6760-M.

Hummer, D.G. and Mihalas, D.M. 1988, Ap. J., 331, 794.

Iglesias, C.A., Rogers, F.J. and Wilson, B.G. 1987, Ap. J.(letters), 322, L45. 1990, Ap. J., 360, 221.

Mihalas, D.M., Däppen, W. and Hummer, D.G. 1988, Ap. J., 331, 815.

Seaton, M.J. 1987, J. Phys. B: At. Mol. Phys., 20, 6363.

Simon, N.R. 1982, Ap. J., 260, L87.

Yu Yan 1991, Rev. Mexicana Astron. Astrof., in print.

\subsection{SESSION 4. APPLICATIONS - Chair: S. Sahal-Bréchot}

\author{
NEW OPACITIES \\ Robert L. KURUCZ \\ Harvard-Smithsonian Center for Astrophysics, 60 Garden Street, Cambridge, MA 02138, U.S.A.
}

I have described my atomic and molecular line data calculations at the IAU general Assembly in Baltimore (Kurucz 1988) and at a NATO workshop in Trieste (Kurucz 1991a). I now have data for 58 million atomic and diatomic molecular lines.

The opacities are calculated with a version of my spectrum synthesis computer program that has been under development since 1965 and has been described by Kurucz and Furenlid (1981) and by Kurucz and Avrett (1981). The algorithms for computing the total line opacity are extremely fast because maximum use is made of temperature and wavelength factorization and pretabulation. There is no limit to the number of spectrum lines that can be treated. The equation of state and continuum opacities are computed with a version of the model atmosphere program ATLAS (Kurucz 1970). Photoionization continua are put in at their exact positions, each with its own cross-section and with the series of lines that merge into each continuum included so that there are no discontinuities in the spectrum. Hydrogen line profiles are computed using a routine from Peterson (1979). Autoionization lines have Shoreparameter Fano profiles. Other lines have Voigt profiles that are computed accurately for any value of the damping parameter $a$ which includes radiative, Stark, and van der Waals broadening.

In late 1988 I used the line data to compute new solar abundance opacity tables for use in my stellar atmosphere modelling. The calculations included all $58,000,000$ lines for 56 temperatures from $2000 \mathrm{~K}$ to $200000 \mathrm{~K}, 21 \mathrm{log}$ pressures from -2 to +8 , and 5 microturbulent 
velocities $0,1,2,4,8 \mathrm{~km} / \mathrm{s}$, and took a large amount of Cray time at the San Diego Supercomputer Center. The spectral resolution was 500000 for 3,500,000 wavelength points in the wavelength range is $8.97666 \mathrm{~nm}$ to $10000 \mathrm{~nm}$. The opacity is tabulated both as 12-step distribution functions for intervals on the order of 1 to $10 \mathrm{~nm}$, and as opacity sampling where, simply, every hundredth wavelength point in the calculation was saved. There are actually two sets of distribution functions: a higher resolution version with 1212 "little" intervals, and a lower resolution version with 328 "big" intervals. The "little" wavelength intervals are nominally $1 \mathrm{~nm}$ in the ultraviolet and $2 \mathrm{~nm}$ in the visible.

I have rewritten my model atmosphere program to use the new line opacities, additional continuous opacities, and an approximate treatment of convective overshooting. The opacity calculation was checked by computing a new solar model (Kurucz 1991b) that matches the observed irradiance (Neckel and Labs 1984; Labs et al. 1987). I am confident that I have solved the missing opacity problem.

Since the beginning of 1990 I have been able to take tremendous advantage of the new Cray YMP at the San Diego Supercomputer Center. In a few months I ran more than I had expected to do in two years. I computed opacities ranging from 0.00001 solar to 10 times solar, enough to compute model atmospheres ranging from the oldest Population II stars to high abundance Am (metallic line) and Ap (peculiar) stars. The hardest part was transmitting the results (200 tapes) back to Cambridge over Internet, but even that usually worked quite well. The exact abundances are $[+1.0],[+0.5],[+0.3],[+0.2],[+0.1],[+0.0],[-0.1],[-0.2],[-0.3],[-0.5],[-1.0],[-1.5],[-2.0],[-$ $2.5],[-3.0],[-3.5],[-4.0],[-4.5],[-5.0],[+0.0$, no He]. The final files for each abundance require two 6250 bpi VAX backup tapes. I have begun to distribute copies of the tapes. I plan to produce 600 megabyte CD-ROMs of these opacities that can be read on any workstation with a CD reader.

Rosseland mean opacities have been computed for each abundance for temperatures up to $200000 \mathrm{~K}$ for use in interiors and envelope calculations. The mass density and electron number were also saved to allow changing variables. These Rosseland opacities should be much better than the Los Alamos opacities in the same temperature region because they treat all the lines explicitly and with detailed profiles, and because the equation of state and the opacity spectra are computed for the actual composition, not as a post facto mixture. The numerical accuracy of any sort of mean opacity must be more reliable in my calculation.

I am open to suggestions for computing opacities for other abundance mixes. At the present time I am planning to compute $\mathrm{C} / \mathrm{O}$ variations, Population II opacities with enhanced light even elements, and some Am and Ap mixes.

\section{Acknowledgements}

This work is supported in part by NASA grants NSG-7054, NAG5-824, and NAGW-1486, and has been supported in part by NSF grant AST85-18900. The most important contribution to this work is a large grant of Cray computer time at the San Diego Supercomputer Center.

\section{$\underline{\text { References }}$}

Kurucz, R.L., 1970: SAO Spec. Rep. No. 309, 291 pp.

Kurucz, R.L., 1991a: pp.440-448 in Stellar Atmospheres: Beyond Classical Models (ed. by L. Crivellari, I. Hubeny, and D.G. Hummer), NATO ASI Series, Kluwer, Dordrecht.

Kurucz, R.L., 1991b: pp. 27-44 in Precision Photometry: Astrophysics of the Galaxy (ed.

A.G.Davis Philip, A.R. Upgren, and K.A. Janes) L. Davis Press, Schenectady.

Kurucz, R.L. and Avrett, E.H., 1981: SAO Spec. Rep. No. 391, 145 pp.

Kurucz, R.L. and Furenlid, I.,1981: SAO Spec. Rep. No. 387, 142 pp.

Labs, D., Neckel, H., Simon, P.C., Thuillier, G. 1987: Solar Phys ics, 107, 203-209.

Neckel, H. and Labs, D., 1984: Solar Physics, 90, 205-258.

Peterson, D.M., 1979 : Personal communication. 


\section{OPACITIES AND CEPHEID MODELS}

Shashi KANBUR

Department of Physics and Astronomy, 260 Behlen, University of Nebraska, Lincoln, Nebraska, NE 68588, USA.

Beat Cepheids are classical Cepheids which pulsate simultaneously in the fundamental and first overtone. The two periods can be accurately disentangled and extracted from observations. In attempting to analyze such stars, use is made of the Petersen diagram where the ratio of the first overtone to fundamental period, $\mathrm{P} 1 / \mathrm{P} 0$, is plotted against the fundamental period $\mathrm{P} 0$. One approach in modelling such stars is to use linear non-adiabatic theory, with masses and luminosities mandated by stellar evolutionary calculations, to compute the fundamental and first overtone modes.

The theoretical and observed period ratios P1/P0 can then be plotted on the same Petersen diagram. Figure 1 of Moskalik, Buchler and Marom (1991) shows such a diagram. The dashed curves denote models calculated with the existing Los Alamos opacities, whilst the dots denote observations.The figure shows a clear disagreement between observations and theory.

These and other discrepancies between pulsation and evolutionary theory led to the suggestion that the opacities may be in error (Simon 1982). Two groups (Iglesias and Rogers 1987 and Seaton 1987) have now recalculated opacities. Moskalik, Buchler and Marom (1991) have used the OPAL (Iglesias and Rogers 1987). Figure 1 of that paper shows that now the theoretical period ratios lie in the loci of observations. Figure 1 of Simon and Kanbur shows calculations made with the Opacity project opacities (Seaton 1987). Again is good agreement between observations and theory.

Since the OPAL and Opacity Project opacities have been calculated using different techniques, but yield similar (though not exactly the same) results, it seems that the conjecture of Simon (1982) was correct.

Simon and Kanbur (1991) give a fuller description of this another problems in pulsation theory which could be alleviated by the use of both the OPAL and Opacity Project opacities.

\section{References}

Iglesias, C.A. and Rogers, F.J., 1991, Ap.J, 371, 408.

Moskalik, Buchler and Marom, 1991, Ap.J., submitted.

Seaton, M.J., 1987, J.Phys. B., 20, 6363.

Simon, N.R., 1982, Ap.J., 260, L87.

Simon, N.R. and Kanbur, S.M. (1991) Revista Mexicana de Astronomia y Astrofisica.

** *

\section{Open scientific meeting (July 31, session 3)}

\subsection{HIGHLIGHTS AND UPDATE OF THE TRIENNUM REPORT}

\subsubsection{Working Group 1 : Atomic Spectra and Wavelength Standards (Report Update) \\ W. C. MARTIN}

Critical compilations of wavelengths and their energy level classifications have been published for all spectra of $\mathrm{Mg}$ [1] and Al [2], and for $\mathrm{Cu}$ X-XXIX [3].

$\mathrm{O}^{\prime}$ Brian et al. [4] give accurate new values for $442 \mathrm{Fe}$ I levels; many improved wavelengths can be obtained from these levels. Johansson and Learner's new measurements of Fe I infrared lines are available [5], and Nace et al. [6] give new high-accuracy wavelengths for $539 \mathrm{Fe}$ I, II ultraviolet lines. Persson et al. [7] have completed a large extension of the Ne III analysis.

References:

[1] Kaufman, V., Martin W. C. : 1991, J. Chem. Ref. Data, 20, 83-152.and [2], ibidem, 775-858.

[3] Shirai, T., et al. : ibid., 1-81. 
[4] O'Brian, T.R., et al.: 1991, J. Opt. Soc. Am. B 8, 1185-1201.

[5] Johansson, S., Learner, R.C.M.: 1990, Astrophys. J. 354, 755-762.

[6] Nave, G., et al:: 1991, J. Opt. Soc. Am. B 8, 2028-2041.

[7] Persson, W., et al.: 1991, Phys.Rev. A 43, 4791-4823.

\subsubsection{Supplemental Report of Working Group 2 : Atomic Transition Probabilities}

W.L. WIESE

Since the completion of the working group report in the summer of 1990, several significant new papers on atomic transition probability data of astrophysical interest were published and some other developments have occurred that are worth noting.

A comprehensive paper containing transition probabilities for 1800 lines of $\mathrm{Fe} \mathrm{I}$, obtained by combinaison of atomic lifetime and branching ratio measurements, was published by O'Brian et al. (1991). Typically, the data are estimated to be accurate in the range from 5\% to $15 \%$ and they agree very closely with the data in the critical compilation by Fuhr et al. (1988). For lines from highly excited levels the new data set should be more accurate and is therefore recommended.

Bell et al. (1990, 1991), Hibbert et al. (1991) and Biémont et al. (1991) have done a series of sophisticated atomic structure calculations, mainly with the CIV3 code, for numerous multiplets of neutral nitrogen and neutral and singly ionized oxygen. Comparisons with a number of recent experimental data, especially from lifetime measurements indicate that these data should be typically in the 5-10\% uncertainty range.

An additional paper in the series "Atomic Data for Opacity Calculations" has been published by Tully et al. (1990), which contains numerous oscillator strength data for the beryllium sequence. An extensive comparison of these "Opacity Project" data with experimental results has been carried out by Allard et al. (1990) for the three Be-like ions C III, $\mathrm{N}$ IV, and $\mathrm{O} \mathrm{V}$. The experimental results and the opacity data typically agree within $\pm 10 \%$. A similar, even more extensive comparison study is underway in the Data Center on Atomic Transition Probalities at NIST. From hundreds of comparisons involving the carbon, nitrogen, and oxygen atoms as well as various ions of these elements, one can draw the - still preliminary - conclusion that the opacity data are especially good for ions where they agree within $5-10 \%$ with the best lifetime results (the latter are also estimated to be accurate within the same uncertainty range). However, for neutral atoms, especially for N I, differences up to $40 \%$ occur. Thus, for the neutral atoms the above mentioned calculations by Bell, Hibbert, and colleagues - which agree much closer with the best experimental data - are recommended in preference to the opacity results.

Fuhr and Wiese (1990) recently revised and expanded their listings of atomic transition probabilities for the 1990-1991 edition of the CRC Handbook of Chemistry and Physics. Several significant additions and revisions were made, especially for $\mathrm{Ba} \mathrm{I}, \mathrm{Hg} \mathrm{I}, \mathrm{Mo}$ I and the element of the Fe-group.

Finally, we would like to draw attention to the proceedings of a 1990 International Colloquium, Edited by J. E. Hansen, which has been largely on the subject of atomic oscillator strengths. Many recent results are given or referenced, and the principal experimental and theoretical approaches are reviewed.

\section{References:}

1. O'Brian, T. R., M. E. Lawler, J. E. Whaling, W., Brault, J. W.: J. Opt. Soc. Am. B. 8, 1185 (1991).

2. Bell, K. L., Hibbert, A.: J. Phys. B. 23, 2673 (1990).

3. Bell, K. L., Hibbert, A., McLaughlin, B. M., Higgins, K.: J. Phys. B. 24, 2665 (1991).

4. Hibbert, A., Biémont, E., Godefroid, M., Vaeck, N.: Astron. Astrophys., Suppl. Ser. 88, 505 (1991).

5. Biémont, E., Hibbert, A., Godefroid, M., Vaeck, N., Fawcett, B. C.: Astrophys. J. 375, 818 (1991).

6. Tully, J. A., Seaton, M. J., Berrington, K. A.: J. Phys. B. 23, 3811 (1990).

7. Allard, N., Artru, M.-C., Lanz, T., Le Dourneuf, M.: Astron. Astrophys., Suppl. Ser. 84, 563 (1990).

8. Fuhr, J. R., Wiese, W. L.: "Atomic Transition Probalities" CRC Handbook of Chemistry and Physics, (1990-1991 Edition, pages 10-128-179, D. L. Lide, Ed., CRC Press, Boca Raton (1990)).

9. Hansen, J. E., Ed., "Atomic spectra and Oscillator Strengths for Astrophysics and Fusion Research", North-Holland, Amsterdam, (1990). 


\subsubsection{Highlights Working Group 5: Molecular Structure and Transitions Data} W. H. PARKINSON

The highlights of significant achievement occured over the full spectral range and involved both laboratory study and astronomical detection.

The increased activity in spectroscopy of silicon-bearing carbon molecules and of hydrocarbon radicals is indicative of the achievements and discoveries at longer wavelengths. For example, the laboratory measurements by the groups at NIST (Lovas et al. 1989') and at Harvard-Smithsonian (Gottlieb et al. 19892) have led to basic spectroscopic parameters and rotational analysis of the silicon dicarbide molecule ( $\mathrm{Si} \mathrm{CC).}$

Frank Lovas has emphasized (Lovas and Suenram 198933, Lovas 19904) the increase in the number of hydrocarbon radicals observed in the microwave region. Spectacular examples of these are the new laboratory spectroscopic studies of carbon molecules. The first was the work on the carbene ring $\mathrm{C}_{3} \mathrm{H}_{2}$ (Vrtilek et al. $1987^{5}$ ), followed, recently, by the laboratory detection of two molecules in the sequence of linear carbenes, $\mathrm{H}_{2} \mathrm{CCC}$ (Vrtilek et al. 19906), and $\mathrm{H}_{2} \mathrm{CCCC}$ (Killian et al. 19907). Astronomical detection of both of these molecules followed the laboratory observation (Cernicharo et al. $1991^{8}$ ), and Cernicharo et al. 19919).

Infrared observations of auroral activity on Jupiter and of laboratory light sources have provided significant spectroscopic informations of the fundamental astrophysical species, $\mathrm{H}_{3}{ }^{+}$ (eg. Maillard et al. $1990^{10}$ and Oka and Geballe $1990^{11}$ ).

There has been interesting new work in the area of molecular structure and spectroscopy involving studies of high-spin multiplicity systems of $\mathrm{N}_{2}$, never before characterized (Partridge et al. $1988^{12}$ and Huber and Vervloet 1988'3).

Considerable new theoretical and experimental effort has been directed toward obtaining quantitative spectroscopic data for CO in the ultraviolet (Kirby and Cooper 198914, Eidelsberg and Rostas 199015, and Stark et al. 199116).

The predissociation line widths of the Schumann-Runge bands of ${ }^{16} \mathrm{O}_{2},{ }^{18} \mathrm{O}_{3}$, and ${ }^{16} \mathrm{O}^{18} \mathrm{O}$ have been derived from their measured absolute cross sections (Yoshino et al. 198917, 199018, $1990^{19}$.

R.W. Nicholls and colleagues have worked to enhance significantly their spectral synthetis codes which they have recently applied to $\mathrm{O}_{2}$ Herzberg system $\left(1991^{20} ; 1991^{21}\right)$.

Phillips and Davis and colleagues have made significant extension to the analysis and understanding of $\mathrm{CaH}$ and $\mathrm{FeH}\left(1988^{22}\right)$.

\section{References}

(1) Suenram, R.D., Lovas, F.J. and Matsumara, K., 1989, Astrophys. J. Lett. 342, L103.

(2) Gottlieb, C.A., Vrtilek, J.M., and Thaddeus, P., 1989, Astrophys. J. Lett. 349 , L29.

(3) Lovas, F.J., and Suenram, R.D., 1989, J. Chem. Ref. Data 18, 1245.

(4) Lovas, F.J., 1990, Private communication.

(5) Vrtilek, J.M., Gottlieb, C.A., and Thaddeus, P., 1987, Astrophys. J. 314, 716.

(6) Vrtilek, J.M., Gottlieb, C.A., Gottlieb, E.W., Killian, T.C., and Thaddeus, P., 1990, Astrophys. J. Lett. 354, L53.

(7) Killian, T.C., Vrtilek, J.M., Gottlieb, C.A., Gottlieb, E.W., and Thaddeus, P., 1990, Astrophys. J. Lett. 365, L89.

(8) Cernicharo, J., Gottlieb, C.A., Guélin, M., Killian, T.C., Paubert, G., Thaddeus, P., and Vrtilek, J.M., 1991, Astrophys. J. Lett. 368, L39.

(9) Cernicharo, J., Gottlieb, C.A., Guélin, M., Killian, T.C., Thaddeus, P., and Vrtilek, J.M., 1991, Astrophys. J. Lett. 368, L43.

(10) Maillard, J.P., Drossart, P., Watson, J.K.G., Kim, S.J., and Caldwell, J., 1990, Astrophys. J. 363, L37.

(11) Oka, T. and Geballe, T.R., 1990, Astrophys. J. 361, L53.

(12) Partridge, H., Langhoff, S.R., Bauschlicher, C.W., and Schwenke, O.W., 1988, J. Chem. Phys. 88, 3174.

(13) Huber, K.P. and Vervloet, M., 1988, J. Chem. Phys. 89, 5957.

(14) Kirby, K. and Cooper, D.L., 1989, J. Chem. Phys. 90, 4895.

(15) Eidelsberg, M. and Rostas, F., 1990, Astron. Astrophys. 235, 472.

(16) Stark, G., Yoshino, K., Smith, P.L., Ito, K., and Parkinson, W.H., 1991, Astrophys. J. 369, 574.

(17) Freeman, D.E., Cheung, A.S-C., Yoshino, K., and Parkinson, W.H., 1989, J. Chem. Phys. 91, 6538. 
(18) Cheung, A.S-C., Yoshino, K., Esmond, J.R., Chiu, S.S-L., Freeman, D.E., and Parkinson, W.H., 1990, J. Chem. Phys. 92, 842.

(19) Chiu, S.S-L., Cheung, A.S-C., Yoshino, K., Esmond, J.R., Freeman, D.E., and Parkinson, W.H., 1990, J. Chem. Phys. 93, 5539.

(20) Cann, M.W.P., and Nicholls, R.W., 1991, Can. J. Phys. (in press).

(21) Cann, M.W.P., Lui, C.W., and Nicholls, R.W., 1991, JQRST (in press).

(22) Phillips, J.G., and Davis, S.P., 1988, Astrophys. J. Suppl. Ser. 66, 227.

\subsection{CONTRIBUTED PAPERS}

\subsubsection{Accurate oscillator accurate oscillator strengths of astrophysical interest for neutral carbon, nitrogen and oxygen. \\ E. BIÉMONT ${ }^{1}$, A. HIBBERT ${ }^{2}$, M. GODEFROID ${ }^{3}$ AND N. VAECK ${ }^{3}$ \\ 1 Université de Liège, Belgique, ${ }^{2}$ The Queen's University of Belfast, UK, 3 Université Libre de Bruxelles, Belgique}

C, N, O are major volatiles which are incompletely condensed in meteorites. Consequently, the sun remains the basic source of information for the solar system abundances. A detailed consideration of correlation effects and of departures from $L S$-coupling is necessary for solar abundance studies. For these reasons, new transition probabilities based on configuration interaction wavefunctions have been calculated in the dipole length and dipole velocity forms of the matrix elements for allowed and spin-forbidden transitions of $\mathrm{N} \mathrm{I}$ and $\mathrm{O} 1[1,2]$. For $\mathrm{C} I$ the calculations are in progress and will be published soon. In the framework of the Breit-Pauli approximation, configuration interaction was included in the calculations performed with the CIV3 code of Hibbert for all the transitions connecting the $n=3$ and $n=4$ energy levels. In order to improve upon the agreement vetween eigenvalue and experimental energy differences, empirical correstions have been introduced in CIV3.

The accuracy of the transition probalities has been tested from comparisons with the most accurate theoretical or experimental results presently available and can be estimated to be of a few percent.

The results have been used, combined with the best solar data available, to improve the photospheric abundance determination of these elements [3,4]. The final results, considered as the most accurate presently available, are $A_{\mathrm{N}}=7.99 \pm 0.04$ and $A_{\mathrm{O}}=8.86 \pm 0.04$, respectively, in the usual logarithmic scale.

\section{References}

[1] Hibbert, A., Biémont, E., Godefroid, M. and Vaeck, N., 1991, Astron. Astrophys. Suppl. 88, 505

[2] Hibbert, A., Biémont, E., Godefroid, M. and Vaeck, N., 1991, J. Phys. B (in press)

[3] Biémont, E., Froese Fischer, C., Godefroid, M., Vaeck, N., Hibbert, A., 1990, Proceedings of the meeting Atomic Data and Oscillator Strengths for Astrophysics and Fusion Research, Ed. J.E. Hansen, Amsterdam, p.59.

[4] Biémont, E., Hibbert, A., Godefroid, M., Vaeck, N., Fawcett, B.C., 1991, Astrophys. J. 375, 818.

\subsubsection{Spectral study of highly ionized noble gases in high temperature plasmas M. GALLARDO +, J. REYNA ALMANDOS *, F. BREDICE * AND M. RAINERI ** Atomic Spectroscopy Group, Optical Research Center (CIOp), C C 124, (1900) La Plata, Argentina}

The spectra of highly charged ions of NEON, ARGON, KRYPTON and XENON with few valence electrons have been studied in the 300-2100 A range using a pulsed discharge tube. For Ne spectra we found lines up to the sixth degree of ionization, and we found lines corresponding to Ar VIII, $\mathrm{Kr}$ VIII and Xe IX.

In the spectral analysis we used isoelectronic comparisons and atomic calculations. We found new energy levels and classified lines and, in the existing levels values, the uncertainty has been considerably decreased.

+ Research of the CONICET of Argentina., * Research of the C.I.C. of Argentina., "*Fellow of the C.I.C. of Argentina. 


\author{
3.2.3. A new technique for storing and interpolating rate coefficients \\ ALAN BURGESS ${ }^{*}$ AND JOHN A. TULLY ${ }^{\dagger}$ \\ * : D.A.M.T.P., Silver Street, Cambridge CB3 9EW, England. \\ f : Observatoire de la Côte d'Azur, URA 1362 du C.N.R.S., B.P. 139, 06003 Nice Cedex, France.
}

We have developed a powerful technique for compacting and assessing atomic data for electron impact excitation of positive ions. Rate coefficients for such processes are derived from thermally average collision strengths $\Upsilon(T)$ which usually vary smoothly with temperature. The present method begins by scaling $\Upsilon$ in order to produce a reduced form $\Upsilon_{r}$. This is then plotted as a function of the scaled temperature $T_{r}$ which maps the entire range of $T$ onto the interval $(0,1) . \Upsilon_{r}$ can be accurately fitted by a 5-point cubic spline, so economising on storage while at the same time providing a convenient means for interpolating and extrapolating data. Straightforward tabulation normally requires more data points than this to cover a limited temperature range. With increasing amounts of atomic data becoming available, the problem of storage needs careful consideration. The present method is a possible solution. It forms the basis of an interactive computer program called OMEUPS which uses graphical display and is designed to be convenient for use by astrophysicists as well of those working in atomic collision theory. Some graphic examples will be presented to illustrate the method.

\title{
3.2.4. New data for the ground electronic state of molecular hydrogen
}

\author{
M.E. MICKELSON ${ }^{*}$, L.E. LARSON* ${ }^{*}$ D.W. FERGUSON ${ }^{\dagger}$ AND K. NARAHARI RAO ${ }^{\dagger}$ \\ * : (Denison University, Granville Ohio 43023, USA) \\ t : (The Ohio State University, Columbus, Ohio 43210, USA)
}

The precision and accuracy of modern high resolution spectroscopic observations of cosmic bodies continues to drive the need for improved laboratory data and theories of atoms and molecules. Inparticular, recent measurements of asymmetric molecular hydrogen quadrupole lines in the absorption spectrum of Uranus and observations of high $J$ rotational transitions in the infrared emission spectrum of molecular hydrogen in the Orion nebulac point to the need for high resolution and high accuracy data. We have recently completed the measurement of pressure shifts, line strengths and shapes, broadening coefficients and line positions for the $S(0)$ and $S(1)$ lines of molecular hydrogen in the 4-0 vibration-rotation band. We have also detected for the first time in the laboratory the 5-0 S(1) line and confirm its detection in Uranus. With these and other high quality measurements of the overtone spectrum of molecular hydrogen in the infrared and visible, a consistent set of parameters has emerged which should be useful to astrophysicists and may challenge theorists to improve their $\mathrm{ab}$-initio calculations.

\subsubsection{An investigation of Brueckner's theory of line broadening with application to the sodium D-lines \\ S.D. ANSTEE AND B.J. O'MARA \\ Department of Physics, the University of Queensland, St Lucia, Queensland 4072, Australia}

Approximations in Brueckner's theory of spectral broadening by collisions with neutral hydrogen atoms relevant to a solar-type atmosphere have been discussed, and a modified theory for $s-p$ transitions has been presented. The theory utilises explicit expressions for the interatomic interaction energy between a hydrogen atom in its ground state and general $m=0, \pm 1 p$-states, derived from second-order perturbation theory without exchange, allowing for removal of the Lindholm-Foley average over $m$-states in the original Brueckner model. Approximate upper and lower bounds for the linewidth of the sodium D-lines are derived, and these values are contrasted with available theoretical, experimental and solar empirical results. The removal of the Lindholm-Foley average is shown to reduce the D-state linewidths by about $30 \%$, and an analysis of the interatomic separations important in the line-broadening cross-section for the D-lines has shown that there is little atomic overlap at the separations that are important. 